\title{
On Stationary Incompressible Norton Fluids and some Extensions of Korn's Inequality
}

\author{
M. Fuchs
}

\begin{abstract}
A simple mathematical model for a so-called Norton fluid is given. We study a variational problem and make use of appropriate versions of Korn's inequality.
\end{abstract}

Keywords: Non-Newtonian fluids, Korn's inequality

AMS subject classiflcation: 49S05, 76A05

\section{Introduction and statement of the results}

We study a variational problem modelling incompressible Norton fluids (see $[4,6]$ ). Let $\Omega$ denote a bounded region in $\mathbb{R}^{3}$ and suppose that on some part $\Gamma$ of $\partial \Omega$ a function $\phi: \Gamma \rightarrow \mathbb{R}^{3}$ is given. Then we look for a minimizer $u: \Omega \rightarrow \mathbb{R}^{3}$ of $F(u)=\int_{\Omega}|\mathcal{E}(u)|^{p} d x$ subject to the side conditions $\left.u\right|_{\mid}=\phi, \operatorname{div} u=0$ on $\Omega$. Here $\mathcal{E}(u)=\frac{1}{2}\left(\nabla u+(\nabla u)^{T}\right)=$ $\left(\frac{1}{2}\left(\partial_{i} u^{j}+\partial_{j} u^{i}\right)\right)_{1 \leq i, j \leq 3}$ is the symmetric part of the velocity gradient $\nabla u$ and $p$ is a fixed real number in $(1, \infty)$. For $p=2$ we have a Newtonian fluid and it is well known (compare [2]) how to handle the above problem with the help of Korn's inequality. On the other hand the limit case $p=1$ corresponds to functional's with linear growth in $\mathcal{E}(u)$ (or more precisely in $\mathcal{E}^{D}(u)=\mathcal{E}(u)-\frac{1}{3} \operatorname{trace} \mathcal{E}(u) \cdot 1$ ) arising in plasticity theory and leads to the study of variational problems in subclasses of the space $B D(\Omega)$ (we refer the reader to the papers [1] and [7] where one also finds further references). The objective of our paper now is to give the appropriate setting for growth rates $p \in(1, \infty), p \neq 2$, which means to prove versions of Korn's inequality in $H^{1, p}\left(\Omega, \mathbb{R}^{3}\right)$.

From now on we assume that $\Omega$ is a domain of class $C^{3, \alpha}$ for some $0<\alpha<1$ and that $\Gamma$ is an open portion of $\partial \Omega$ with $\mathcal{H}^{2}(\Gamma)>0$. Suppose that $\phi \in H^{1, p}\left(\Omega, R^{3}\right)$ is given such that the class

$$
\mathcal{C}=\left\{u \in H^{1, p}\left(\Omega, \mathbb{R}^{3}\right):\left.u\right|_{\Gamma}=\left.\phi\right|_{\Gamma}, \operatorname{div} u=0 \text { a.e. }\right\}
$$

is non-empty (here we consider $1<p<\infty$ ).

Theorem 1: The variational problem $F(u)=\int_{\Omega}|\mathcal{E}(u)|^{p} d x \rightarrow \min$ in $\mathcal{C}$ admits a (unique) solution $u \in \mathcal{C}$.

M. Fuchs: Universität des Saarlandes, FB 9 Mathematik, Bau 27, D - 66041 Saarbrücken 
Remark: Of course the existence result of Theorem 1 can be extended to variational problems of the form

$$
\int_{\Omega} W(x, \mathcal{E}(u)(x)) d x \rightarrow \min
$$

in the class $\mathcal{C}$ provided the dissipation function $W: \Omega \times\left\{S \in \mathbb{R}^{3 \times 3}: S\right.$ symmetric $\} \rightarrow \mathbb{R}$ is convex with respect to the second argument and of $p$ growth for some power $p \in(1, \infty)$. For example a condition of the form $\lambda|S|^{p} \leq W(x, S) \leq \Lambda|S|^{p}$ (with positive numbers $\lambda$ and $\Lambda$ ) would be sufficient. If $W$ is in addition differentiable with respect to $S$, then also an analogy of the following Theorem 2 is true.

Theorem 2: There is a function $f \in L_{l o c}^{p /(p-1)}(\Omega)$ such that, for the minimizer $u \in \mathcal{C}$ from Theorem 1 , we have

$$
p \int_{\Omega}|\mathcal{E}(u)|^{p-2} \mathcal{E}(u) \mathcal{E}(\psi) d x=\int_{\Omega} \operatorname{div} \psi \cdot f d x
$$

for all $\psi \in C_{0}^{\infty}\left(\Omega, \mathbb{R}^{3}\right)$.

The existence result Theorem 1 follows from the following

Theorem 3 (Korn's inequality): There is a constant $c=c(p, \Omega, \Gamma)$ with the property that

$$
\|v\|_{H^{1, P}(\Omega)} \leq c\|\mathcal{E}(v)\|_{L^{P}(\Omega)}
$$

holds for all $v \in H^{1, p}\left(\Omega, \mathbb{R}^{3}\right)$ such that $\left.v\right|_{\Gamma}=0$.

In Section 2 we prove Korn's inequality with the help of several lemmas, the Euler equation will be discussed in Section 3 .

Accepting Theorem 3 we select a minimizing sequence $\left\{u_{n}\right\} \subset \mathcal{C}$ and deduce

$$
\left\|u_{n}-\phi\right\|_{H^{1, P}(\Omega)} \leq c\left[F\left(u_{n}\right)+F(\phi)\right]^{1 / p}
$$

so that $\sup _{n}\left\|u_{n}\right\|_{H^{1, p}(\Omega)}<\infty$ and, for some element $u, u_{n} \rightarrow u$ in $H^{1, p}\left(\Omega, \mathbb{R}^{3}\right)$ at least for a subsequence. Since $F$ is weakly lower semicontinuous $F(u) \leq \inf _{\mathcal{c}} F$. Clearly $\left.u\right|_{\Gamma}=\phi$ and $\operatorname{div} u=0$ follows from

$$
\int_{\Omega} \operatorname{div} u \cdot \eta d x=\lim _{n \rightarrow \infty} \int_{\Omega} \operatorname{div} u_{n} \cdot \eta d x=0
$$

for all $\eta \in C_{0}^{\infty}(\Omega)$, hence $u$ is minimizing. If $\tilde{u} \in \mathcal{C}$ is also minimizing, then we must have $\mathcal{E}(u)=\mathcal{E}(\tilde{u})$ and from Theorem 3 we infer $\|u-\tilde{u}\|_{H^{1, p}(\Omega)}=0$. This proves Theorem 1 . 


\section{Korn's inequality in the space $H^{1, p}\left(\Omega, R^{3}\right), 1<p<\infty$}

We here assume that $\Omega \subset \mathbb{R}^{3}$ is a domain satisfying the assumptions of [8: Satz I.5.2].

Lemma 1: There exists a positive constant $c=c(p, \Omega)$ with the following property: If $f \in L^{p}(\Omega)$ satisfies $\int_{\Omega} f d x=0$, then we find a vector field $U \in \stackrel{\circ}{H}^{1, p}\left(\Omega, \mathbb{R}^{3}\right)$ such that $\operatorname{div} U=f$ on $\Omega$ and $\|U\|_{H^{1, p}(\Omega)} \leq c\|f\|_{L^{p}(\Omega)}$.

Remark: 1. The strong smoothness properties imposed on $\partial \Omega$ enter our arguments only in the proof of Lemma 1 . So Theorems $1-3$ can be extended to precisely those class of bounded domains $\Omega \subset \mathbb{R}^{3}$ for which Lemma 1 is true. 2. For balls $\Omega$ we have $c=c(p)$.

Proof of Lemma 1: We select a sequence of functions $f_{n} \in C_{0}^{\infty}(\Omega)$ such that $f_{n} \rightarrow$ $f$ in $L^{p}(\Omega)$. According to [8: Satz I.5.2] there exists $U_{n} \in C^{1, \alpha}\left(\Omega, R^{3}\right) \cap C^{0, \alpha}\left(\bar{\Omega}, \mathbb{R}^{3}\right)$ such that

$$
\operatorname{div} U_{n}=f_{n}-\left(f_{n}\right)_{\Omega} \text { on } \Omega,\left.\quad U_{n}\right|_{\partial \Omega}=0, \quad\left\|\nabla U_{n}\right\|_{L^{P}(\Omega)} \leq c\left\|f_{n}-\left(f_{n}\right)_{\Omega}\right\|_{L^{p}(\Omega)}
$$

for a constant $c$ as in Lemma 1. Hence $U_{n} \in H^{1, p}\left(\Omega, \mathbb{R}^{3}\right)$ and [5: Theorems 3.6.2 and 3.6.3] imply $U_{n} \in \stackrel{\circ}{H}^{1, p}\left(\Omega, \mathbb{R}^{3}\right)$. From the above estimate we deduce that $\left\{U_{n}\right\}$ is a Cauchy sequence in $\stackrel{\circ}{H}^{1, p}\left(\Omega, \mathbb{R}^{3}\right)$ and for the limit $U$ we obtain the equation $\operatorname{div} U=$ $f-(f)_{\Omega}=f$. Here and in the following $(f)_{\Omega}=f_{\Omega} f d x$ denotes the mean value

Lemma 2: For $\Omega$ as above and $1<q<\infty$ consider a distribution $T: C_{0}^{\infty}(\Omega) \rightarrow \mathbb{R}$ with the property $T, \partial_{i} T \in\left(\stackrel{\circ}{H}^{1, q}(\Omega)\right)^{*}$ for $i=1,2,3$ where * means the dual space, i.e.

$$
|T(\varphi)|+\sum_{i=1}^{3}\left|T\left(\partial_{i} \varphi\right)\right| \leq C\|\varphi\|_{H^{1, \varphi}(\Omega)} \quad \text { for all } \varphi \in C_{0}^{\infty}(\Omega) .
$$

Then $T(\varphi)=\int_{\Omega} u \cdot \varphi d x$ for a function $u \in L^{q^{\prime}}(\Omega), q^{\prime}=q /(q-1)$.

Proof: Let $G$ denote a subregion of $\Omega$ with the same regularity properties. For $\varepsilon<\varepsilon_{0}, \varepsilon_{0}=\operatorname{dist}(G, \partial \Omega)$, we let $\Phi_{\varepsilon}(z):=\varepsilon^{-3} \Phi\left(\frac{z}{\varepsilon}\right) \in C_{0}^{\infty}\left(B_{\varepsilon}(0)\right)$ for a mollifier $\Phi \in C_{0}^{\infty}\left(B_{1}(0)\right)$. Then, according to [3], for $\varphi \in C_{0}^{\infty}(G)$ we have

$$
T_{\varepsilon}(\varphi):=T\left(\Phi_{\epsilon} * \varphi\right)=\int_{G} \varphi(y) T_{x}\left(\Phi_{\varepsilon}(x-y)\right) d y \quad \text { and } \quad \lim _{\varepsilon \downarrow 0} T\left(\Phi_{\varepsilon} * \varphi\right)=T(\varphi)
$$

$\left(\right.$ clearly $\operatorname{spt}\left(x \mapsto \Phi_{e}(x-y)\right) \subset \Omega$ for arbitrary $y \in G$ so that $u_{e}(y):=T_{x}\left(\Phi_{e}(x-y)\right)$ is well defined). We have

$$
\begin{aligned}
\left|\int_{G} \partial_{i} \varphi \cdot u_{e} d x\right| & =\left|T\left(\Phi_{e} * \partial_{i} \varphi\right)\right| \\
& =\left|T\left(\partial_{i}\left(\Phi_{\varepsilon} * \varphi\right)\right)\right|=\left|\left(\partial_{i} T\right)\left(\Phi_{e} * \varphi\right)\right| \\
& \leq C\left\|\Phi_{\varepsilon} * \varphi\right\|_{H^{1, q}(\Omega)} \leq C\|\varphi\|_{H^{1, q}(G)} \quad\left(\varphi \in C_{0}^{\infty}(G)\right) .
\end{aligned}
$$

For the last inequality one has to observe

$$
\operatorname{spt}\left(\Phi_{\varepsilon} * \varphi\right) \subset G_{\varepsilon}=\{x: \operatorname{dist}(x, G)<\varepsilon\}
$$


which implies $\left\|\Phi_{\epsilon} * \varphi\right\|_{H^{1, q\left(G_{\varepsilon}\right)}} \leq\|\varphi\|_{H^{1, q}\left(G_{2 \varepsilon}\right)}$ by a standard property of mollifiers. But obviously $\|\varphi\|_{H^{1, \varphi\left(G_{2 \varepsilon}\right)}}=\|\varphi\|_{H^{1,9(G)}}$.

By approximation

$$
\left|\int_{G} u_{\varepsilon} \cdot \partial_{i} \varphi d x\right| \leq C\|\varphi\|_{H^{1, q}(G)}
$$

extends to all $\varphi \in \stackrel{\circ}{H}^{1, q}(G)$. Consider $f \in L^{q}(G)$ such that $(f)_{G}=0$. Lemma 1 yields the existence of a field $\psi \in \stackrel{\circ}{H}^{1, q}\left(G, \mathbb{R}^{3}\right)$ such that $\operatorname{div} \psi=f$ and

$$
\|\psi\|_{H^{1, q}(G)} \leq c\|f\|_{L^{q}(G)}, \quad c=c(q, G) .
$$

This implies

$$
\left|\int_{G} u_{e} \cdot f d x\right|=\left|\int_{G} u_{\varepsilon} \operatorname{div} \psi d x\right| \leq c\|f\|_{L^{\natural}(G)}
$$

for all $f \in L^{q}(G)$ with vanishing mean value or

$$
\left|\int_{G}\left(u_{\varepsilon}-\left(u_{e}\right)_{G}\right) \cdot f d x\right| \leq c\|f\|_{L^{\natural}(G)} \quad\left(f \in L^{q}(G)\right)
$$

(observe that $\int_{G}\left(u_{\varepsilon}-\left(u_{\varepsilon}\right)_{G}\right) \cdot f d x=\int_{G} u_{\varepsilon} \cdot\left(f-(f)_{G}\right) d x$ and $\left\|f-(f)_{G}\right\|_{L^{q}(G)} \leq$ $\left.2\|f\|_{L^{q}(G)}\right)$. If we take the supremum over all $f \in L^{q}(G)$ with $\|f\|_{L^{q}(G)} \leq 1$, we have shown

$$
\left\|u_{e}-\left(u_{\varepsilon}\right){ }_{G}\right\|_{L^{\prime}(G)} \leq c(q, G)<\infty
$$

for all sinall $\varepsilon>0$, and there exists $v^{G} \in L^{q^{\prime}}(G)$ such that

$$
u_{\varepsilon}-\left(u_{\varepsilon}\right)_{G} \rightarrow v^{G} \quad \text { in } L^{q^{\prime}}(G) \text { as } \varepsilon \downarrow 0 .
$$

Now we fix some small ball $B_{e}\left(x_{0}\right) \subset G$ and pick

$$
\varphi_{1} \in C_{0}^{\infty}\left(B_{Q}\left(x_{0}\right),[0,1]\right) \quad \text { with } \quad \int_{\mathcal{B}_{\mathfrak{q}}\left(x_{0}\right)} \varphi_{1} d x=1
$$

From

$$
T\left(\varphi_{1}\right)=\lim _{\varepsilon \downarrow 0} \int_{G} u_{e} \cdot \varphi_{1} d x=\lim _{e \downarrow 0}\left\{\int_{G} \varphi_{1} \cdot\left(u_{e}-\left(u_{\varepsilon}\right)_{G}\right) d x+\left(u_{e}\right)_{G}\right\}
$$

we deduce the existence of $\xi^{G}:=\lim _{\varepsilon \downarrow 0}\left(u_{\varepsilon}\right)_{G}$ with value $T\left(\varphi_{1}\right)-\int_{G} v^{G} \cdot \varphi_{1} d x$ and since

$$
T(\varphi)=\lim _{e \downarrow 0}\left\{\int_{G} \varphi \cdot\left(u_{e}-\left(u_{e}\right)_{G}\right) d x+\left(u_{e}\right)_{G} \int_{G} \varphi d x\right\}
$$

holds for arbitrary $\varphi \in C_{0}^{\infty}(G)$ we end up with the representation

$$
T(\varphi)=\int_{G} u^{G} \cdot \varphi d x \quad \text { where } u^{G}=v^{G}+\xi^{G} \in L^{q^{\prime}}(G)
$$

being valid on the space $C_{0}^{\infty}(G)$. The inclusions $G \subset G^{\prime} \subset \Omega$ clearly imply the equality $u^{G}=u^{G^{\prime}}$ almost everywhere on $G$. 
Observe next that

$$
\begin{aligned}
\left\|u^{G}\right\|_{L^{q^{\prime}}(G)} & \leq\left\|v^{G}\right\|_{L^{q^{\prime}}(G)}+\left|\xi^{G}\right| \mathcal{L}^{3}(G)^{1 / q^{\prime}} \\
& \leq c(q, G)+\mathcal{L}^{3}(G)^{1 / q^{\prime}}\left\{\left|T\left(\varphi_{1}\right)\right|+\int_{G}\left|v^{G}\right| \cdot \varphi_{1} d x\right\} \\
& \leq c(q, G)+\mathcal{L}^{3}(G)^{1 / q^{\prime}}\left\{C\left\|\varphi_{1}\right\|_{H^{1, q}\left(B_{p}\left(x_{0}\right)\right)}+\left\|v^{G}\right\|_{L^{\prime}(G)}\left\|\varphi_{1}\right\|_{L^{q}\left(B_{q}\left(x_{0}\right)\right)}\right\} .
\end{aligned}
$$

Here the constant $c(q, G)$ has the form $c C$ with $c$ from Lemma 1 and $C$ denotes the bound for $T, \partial_{i} T$ in $\left(\stackrel{\leftrightarrow}{H}^{1, q}(\Omega)\right)^{*}$.

In a final step we replace $G$ by an increasing sequence $\left\{G_{n}\right\}$ of regular domains exhausting $\Omega$ and define $u \in L_{\text {loc }}^{q^{\prime}}(\Omega)$ through $u(x)=u^{G_{n}}(x)$ if $x \in G_{n}$. By construction $u$ represents the distribution $T$, moreover $c\left(q, G_{n}\right)$ is bounded independent of $n$ so that $u \in L^{q^{\prime}}(\Omega)$ on account of the above estimates

Now we are in the position to prove versions of Korn's inequality.

Lemma 3: For $1<p<\infty$ and $\Omega \subset \mathbb{R}^{3}$ as in Lemma 1 there exists a constant $c(p, \Omega)$ such that

$$
\|u\|_{H^{1, p}(\Omega)} \leq c(p, \Omega)\left[\|u\|_{L^{p}(\Omega)}+\|\mathcal{E}(u)\|_{L^{p}(\Omega)}\right]
$$

for all $u \in H^{1, p}\left(\Omega, \mathbb{R}^{3}\right)$.

Corollary: Consider the Banach space

$$
V=\left\{u \in L^{p}\left(\Omega, \mathbb{R}^{3}\right): \mathcal{E}_{i j}(u)=\frac{1}{2}\left(\partial_{i} u^{j}+\partial_{j} u^{i}\right) \in L^{p}(\Omega)(i, j,=1,2,3)\right\}
$$

equipped with the norm

$$
\|u\|_{V}=\|u\|_{L^{P}(\Omega)}+\|\mathcal{E}(u)\|_{L^{P}(\Omega)}
$$

where $\mathcal{E}_{i j}(u)$ is defined in the sense of distributions. Then $V=H^{1, p}\left(\Omega, R^{3}\right)$ and the norms $\|\cdot\| V$ and $\|\cdot\|_{H^{1, p(\Omega)}}$ are equivalent.

Proof: Consider the continuous embedding $I: H^{1, p}\left(\Omega, \mathbb{R}^{3}\right) \ni u \mapsto u \in V$ and take $v \in V$. Then in the weak sense

$$
\partial_{j} \partial_{k} v^{i}=\partial_{j} \mathcal{E}_{i k}(v)+\partial_{k} \mathcal{E}_{i j}(v)-\partial_{i} \mathcal{E}_{j k}(v) \quad(i, j, k=1,2,3)
$$

Since we assume $\mathcal{E}(v) \in L^{p}\left(\Omega, \mathbb{R}^{3 \times 3}\right)$ the above relation yields $\partial_{j} \partial_{k} v^{i} \in\left(\stackrel{H}{H}^{1, p^{\prime}}(\Omega)\right)^{*}$ (where ${ }^{*}$ indicates the dual space) and $v \in L^{p}\left(\Omega, \mathbb{R}^{3}\right)$ implies $\partial_{k} v^{i} \in\left(\stackrel{H}{ }^{1, p^{\prime}}(\Omega)\right)^{*}$ so that $\partial_{k} v^{i} \in L^{p}(\Omega)$ by Lemma 2 , that is $v \in H^{1, p}\left(\Omega, \mathbb{R}^{3}\right)$ which shows surjectivity of the embedding $I$. Hence $V=H^{1, p}\left(\Omega, \mathbb{R}^{3}\right)$ and the desired estimate follows from the closed graph theorem, i.e. the continiuty of $I^{-1}$

We now come to the 
Proof of Theorem 3: According to Lemma 3 it remains to show

$$
\|v\|_{L^{P}(\Omega)} \leq c\|\mathcal{E}(v)\|_{L^{P}(\Omega)}
$$

for a suitable constant $c=c(p, \Omega, \Gamma)$ and all $v \in H^{1, p}\left(\Omega, R^{3}\right)$ with $v \mid \Gamma=0$. We assume that the statement is wrong, hence there is a sequence $\left\{v_{n}\right\}$ in $H^{1, p}\left(\Omega, \mathbb{R}^{3}\right)$ with $\left.v_{n}\right|_{\Gamma}=0$ such that, without loss of generality, $\left\|v_{n}\right\|_{L^{p}(\Omega)}=1$ and $1>n\left\|\mathcal{E}\left(v_{n}\right)\right\|_{L^{p}(\Omega)}$, i.e. $\mathcal{E}\left(v_{n}\right) \rightarrow 0$ in $L^{p}\left(\Omega, \dot{R}^{3 \times 3}\right)$ as $n \rightarrow \infty$. Quoting Lemma 3 we have $v_{n} \rightarrow v$ in $H^{1, p}\left(\Omega, R^{3}\right)$ (at least for a subsequence) with $v$ satisfying $\|v\|_{L^{p}(\Omega)}=1, v \mid \Gamma=0$ and $\mathcal{E}(v)=0$ (by the weak lower semicontinuity of $\|\mathcal{E}(\cdot)\|_{L^{p}(\Omega)}$ ). On the other hand we know $H^{1, p}\left(\Omega, \mathbb{R}^{3}\right) \subset B D(\Omega)$ so that [1: Corollary 1.11] implies

$$
\|w\|_{L^{3 / 2}(\Omega)} \leq c \int_{\Omega}|\mathcal{E}(w)| d x=0
$$

hence $w=0$ contradicting $\|w\|_{L^{p}(\Omega)}=1$

\section{The existence of a pressure function}

Suppose that $u \in \mathcal{C}$ is the minimizer obtained in Theorem 1. For a suitable field $U \in L^{p^{\prime}}\left(\Omega, \mathbb{R}^{3 \times 3}\right)$ we have

$$
p \int_{\Omega}|\mathcal{E}(u)|^{p-2} \mathcal{E}(u) \mathcal{E}(\psi) d x=\int_{\Omega} U \nabla \psi d x
$$

on the space $\stackrel{\circ}{H}^{1, p}\left(\Omega, \mathbb{R}^{3}\right)$, especially $\int_{\Omega} U \nabla \psi d x=0$ if $\operatorname{div} \psi=0$. Consider a region $G$ as in the proof of Lemma 2; for $\varepsilon<\operatorname{dist}(G, \partial \Omega)$ we define $U_{e}=\Phi_{e} * U$. Then

$$
\int_{\Omega} U_{e} \nabla \psi d x=\int_{\Omega} U \nabla\left(\Phi_{e} * \psi\right) d x=0
$$

for all $\psi \in C_{0}^{\infty}\left(G, \mathbb{R}^{3}\right)$ with $\operatorname{div} \psi=0$, since $\operatorname{div}\left(\Phi_{\varepsilon} * \psi\right)=\Phi_{\varepsilon} * \operatorname{div} \psi$ and $\operatorname{spt}\left(\Phi_{e} * \psi\right) \subset \Omega$. Let $h_{\varepsilon}$ denote the unique element in $\stackrel{\circ}{H}^{1,2}\left(G, \mathbb{R}^{3}\right)$ representing $U_{\varepsilon}$ with respect to the Dirichlet scalar product, i.e..

$$
\left\langle h_{\varepsilon}, \psi\right\rangle:=\int_{G} \nabla h_{e} \nabla \psi d x=\int_{G} U_{e} \nabla \psi d x \quad\left(\psi \in \stackrel{\circ}{H}^{1,2}\left(\Omega, \mathbb{R}^{3}\right)\right) .
$$

Then the above calculations show that this element $h_{\varepsilon}$ is orthogonal to the kernel of the operator $\operatorname{div}: \stackrel{\circ}{H}^{1,2}\left(G, \mathbb{R}^{3}\right) \rightarrow L^{2}(G)$, hence there exists $f_{\epsilon} \in L^{2}(G)$ such that $-\triangle h_{e}=\nabla f_{e}$ which means

$$
\int_{G} U_{\varepsilon} \nabla \psi d x=\int_{G} f_{\varepsilon} \cdot \operatorname{div} \psi d x \quad\left(\psi \in C_{0}^{\infty}\left(G, \mathbb{R}^{3}\right)\right) .
$$

Without changing the above identity we may supppse $\left(f_{e}\right)_{G}=0$. Next we select $g \in$ $C_{0}^{\infty}(G)$ and choose $\psi \in C^{1, \alpha}\left(G, \mathbb{R}^{3}\right) \cap \mathbb{A}^{\rho, \alpha}\left(\bar{\Omega}, \mathbb{R}^{\beta}\right)_{\text {such }}$ that

$$
\left.\psi\right|_{\partial G}=0, \quad \operatorname{div} \psi=g-(g)_{G}, \quad\|\nabla \psi\|_{L^{P}(G)} \leq c\left\|g-(g)_{G}\right\|_{L^{P}(G)} \leq c\|g\|_{L^{P}(G)} .
$$


Then $\left(f_{e}\right)_{G}=0$ yields

$$
\begin{aligned}
\int_{G} f_{e} \cdot g d x & =\int_{G} f_{e} \cdot\left(g-(g)_{G}\right) d x=\int_{G} f_{e} \cdot \operatorname{div} \psi d x \\
& =\int_{G} U_{e} \nabla \psi d x \leq c\|U\|_{L^{p^{\prime}}(\Omega)}\|g\|_{L^{p}(G)}
\end{aligned}
$$

which implies $\left\|f_{e}\right\|_{L^{p^{\prime}}(G)} \leq c(p, G)<\infty$ independent of $\varepsilon$. After passing to the limit we find $f_{G} \in L^{p^{\prime}}(G)$ such that $f_{\varepsilon} \rightarrow f_{G}$ weakly in $L^{p^{\prime}}(G)$ and

$$
\int_{\Omega} U \nabla \psi d x=\int_{G} f_{G} \cdot \operatorname{div} \psi d x \quad \text { for all } \psi \in C_{0}^{\infty}\left(G, \mathbb{R}^{3}\right) .
$$

As before let $\left\{G_{n}\right\}$ denote an increasing sequence of domains such that $\bigcup_{n=1}^{\infty} G_{n}=\Omega$. For each $n \in \mathbb{N}$ we take a function $f_{n}$ satisfying (1) on $G=G_{n}$ (note that (1) fixes $f_{G}$ only up to an additive constant). Then $f_{n+1}-f_{n} \equiv a_{n}$ on $G_{n}$, hence the definition

$$
f(x)= \begin{cases}f_{1}(x) & \text { for } x \in G_{1} \\ f_{2}(x)-a_{1} & \text { for } x \in G_{2} \\ \vdots & \\ f_{n}(x)-\sum_{k=1}^{n-1} a_{k} & \text { for } x \in G_{n}\end{cases}
$$

leads to a well defined function $f \in L_{l o c}^{p^{\prime}}(\Omega)$ satisfying (1) on $\Omega$, i.e. for all $\psi \in$ $C_{0}^{\infty}\left(\Omega, \mathbb{R}^{3}\right)$. From our construction we deduce

$$
\left\|f_{n}\right\|_{L^{p^{\prime}}\left(G_{n}\right)} \leq c\left(p, G_{n}\right)
$$

$c\left(p, G_{n}\right)$ defined in Lemma 1 and bounded independent of $n$.

\section{References}

[1] Anzellotti, G. and M. Giaquinta: Existence of the displacement field for an elasto-plastic body subject to Hencky's law and Von Mises yield condition. Manus. Math. 32 (1980), $101-136$.

[2] Duvaut, G. and J. L. Lions: Inequalities in Mechanics and Physics (Springer Grundlehren: Vol. 219). Berlin - Heidelberg - New York: Springer-Verlag 1976.

[3] Federer, H.: Geometric Measure Theory (Springer Grundlehren: Vol. 153). Berlin Heidelberg - New York: Springer-Verlag 1969.

[4] Tallec, L.: Numerical Analysis of Viscoplastic Problems. Masson: Springer-Verlag 1990.

[5] Morrey jr., C. B.: Multiple Integrals in the Calculus of Variations (Springer Grundlehren: Vol. 130). Berlin - Heidelberg - New York: Springer-Verlag 1966.

[6] Norton, M.: The Creep of Steel at High Temperature. New York: Mac-Graw Hill 1929.

[7] Temam, M. and G. Strang: Functions of bounded deformation. Ann. Rat. Mech. Anal. 75 (1980), 7 - 21.

[8] Wahl, W. von: Vorlesung über das Außennaumproblem für die instationären Gleichungen von Navier Stokes. Vorlesungsreihe SFB 256 Bonn No. 11 (1989), 1 - 371. 